\title{
Parallel iterative linear solvers for multistep Runge-Kutta methods
}

\author{
Eleonora Messina ${ }^{\mathrm{a}}$, Jacques J.B. de Swart ${ }^{\mathrm{b}, *, 1}$, Wolter A. van der Veen ${ }^{\mathrm{b}, 1}$

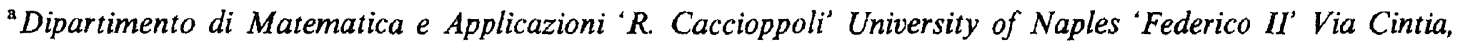 \\ I-80126 Naples, Italy \\ ${ }^{\mathrm{b}}$ CWI, P. O. Box 94079, 1090 GB Amsterdam, The Netherlands
}

Received 20 September 1996; received in revised form 20 July 1997

\begin{abstract}
This paper deals with solving stiff systems of differential equations by implicit Multistep Runge-Kutta (MRK) methods. For this type of methods, nonlinear systems of dimension $s d$ arise, where $s$ is the number of Runge-Kutta stages and $d$ the dimension of the problem. Applying a Newton process leads to linear systems of the same dimension, which can be very expensive to solve in practice. With a parallel iterative linear system solver, especially designed for MRK methods, we approximate these linear systems by $s$ systems of dimension $d$, which can be solved in parallel on a computer with $s$ processors. In terms of Jacobian evaluations and LU-decompositions, the $k$-step $s$-stage MRK applied with this technique is on $s$ processors equally expensive as the widely used $k$-step Backward Differentiation Formula on 1 processor, whereas the stability properties are better than that of BDF. A simple implementation of both methods shows that, for the same number of Newton iterations, the accuracy delivered by the new method is higher than that of BDF.
\end{abstract}

Keywords: Numerical analysis; Newton iteration; Multistep Runge-Kutta methods; Parallelism

AMS classification: Primary: 65L05; Secondary: 65F05; 65F50

\section{Introduction}

For solving the stiff initial value problem (IVP)

$$
y^{\prime}(t)=f(y(t)), \quad y\left(t_{0}\right)=y_{0}, \quad y, f \in \mathbb{R}^{d}, \quad t_{0} \leqslant t \leqslant t_{e},
$$

a widely used class of methods is that of the backward differentiation formulae (BDFs)

$$
y_{n}=\left(\kappa^{\mathrm{T}} \otimes I\right) y^{(n-1)}+h_{n} \beta f\left(y_{n}\right) \text {. }
$$

\footnotetext{
* Corresponding author. E-mail: jacques@cwi.nl

${ }^{1}$ Supported by Dutch Technology Foundation STW, grant no. CWI22.2703.

0377-0427/97/\$17.00 (C) 1997 Elsevier Science B.V. All rights reserved PII S 0377-0427(97)00135-0
} 
Here, $\otimes$ denotes the Kronecker product, the vector $y^{(n-1)}$ is defined by $\left(y_{n-k}^{\mathrm{T}}, \ldots, y_{n-1}^{\mathrm{T}}\right)^{\mathrm{T}}$, where $y_{j}$ approximates the solution at $t=t_{j}$ and $k$ is the number of previous step points that are used for the computation of the approximation in the current time interval. The stepsize $t_{n+1}-t_{n}$ is denoted by $h_{n}$. The scalar $\beta$ and the $k$-dimensional vector $\kappa$ contain the method parameters. They depend on $h^{(n)}$, which is the vector with $k$ previous stepsizes defined by $h^{(n)}:=\left(h_{n-k+1}, \ldots, h_{n}\right)^{\mathrm{T}}$. In the sequel, $I$ stands for the identity matrix and $e_{i}$ for unit vector in the $i$ th direction. The dimensions of $I$ and $e_{i}$ may vary, but will always be clear from the context. For example, the popular codes DASSL [16] and VODE [2] are based on BDFs. A drawback of BDFs is the loss of stability if the number of step points $k$ increases. As a consequence of Dahlquist's order of barrier, no A-stable BDF can exceed order 2. Moreover, BDFs are not zero-stable for $k>6$.

A promising class of methods that can overcome these drawbacks of BDFs are the multistep Runge-Kutta (MRK) methods, which are of the form

$$
y_{n}=\left(\chi^{\mathrm{T}} \otimes I\right) y^{(n-1)}+h_{n}\left(\alpha^{\mathrm{T}} \otimes I\right) F\left(Y_{n}\right)
$$

where $Y_{n}$ is the solution of the equation

$$
R\left(Y_{n}\right)=0, \quad R\left(Y_{n}\right):=Y_{n}-(G \otimes I) y^{(n-1)}-h_{n}(A \otimes I) F\left(Y_{n}\right) .
$$

Here, $Y_{n}$ is the so-called stage vector of dimension $s d$, whose components $Y_{n i}$ represent approximations to the solution at $t=t_{n-1}+c_{i} h_{n}$, where $c:=\left(c_{1}, \ldots, c_{s}\right)^{\mathrm{T}}$ is the vector of abscissae and $s$ is the number of Runge-Kutta stages. The vector $F\left(Y_{n}\right)$ contains the derivative values $f\left(Y_{n i}\right)$. The vectors $\alpha$ and $\chi$, and the matrices $A$ and $G$ contain method parameters and are of dimension $s \times 1, k \times 1, s \times s$ and $s \times k$, respectively. These parameters and the abscissae $c_{i}$ depend on $h^{(n)}$. We remark that a way of circumventing this dependence on $h^{(n)}$ is interpolating the previous step points, so that they are equally spaced. However, this strategy adds local errors and does not allow good stepsize flexibility, see $[17$, p. 68$]$.

Stability of MRKs has been investigated for fixed stepsizes in the literature. Even for large values of $k$, these methods have 'surprisingly' good stability properties [10, p. 296]. For example, MRKs of Radau type with $s=3$ remain stiffly stable for $k \leqslant 28$ and have modest error constants [17, p. 13].

A drawback of using MRKs is the high cost of solving the nonlinear system (3) of dimension $s d$ every time step. Normally, one uses a (modified) Newton process to solve this non-linear system. This leads to a sequence of iterates $Y_{n}^{(0)}, Y_{n}^{(1)}, \ldots, Y_{n}^{(m)}$ which are obtained as solutions of the $s d$-dimensional linear systems

$$
\left(I-A \otimes h_{n} J_{n}\right)\left(Y_{n}^{(j)}-Y_{n}^{(j-1)}\right)=-R\left(Y_{n}^{(j-1)}\right), \quad j=1,2, \ldots, m,
$$

where $J_{n}$ is the Jacobian of the function $f$ in (1) evaluated in $t_{n}$, the starting vector $Y_{n}^{(0)}$ is defined by some predictor formula, and $Y_{n}^{(m)}$ is accepted as approximation to $Y_{n}$. If we use Gaussian elimination to solve these linear systems, then this would cost $\frac{2}{3} s^{3} d^{3}$ arithmetic operations for the LU-decompositions.

In order to reduce these costs, one can bring the Newton matrix $I-A \otimes h_{n} J_{n}$ to block diagonal form by means of similarity transformations [3] resulting in

$$
\begin{aligned}
& \left(I-T^{-1} A T \otimes h_{n} J_{n}\right)\left(X_{n}^{(j)}-X_{n}^{(j-1)}\right)=-\left(T^{-1} \otimes I\right) R\left(Y_{n}^{(j-1)}\right), \\
& Y_{n}^{(j)}=(T \otimes I) X_{n}^{(j)}, \quad j=1,2, \ldots, m .
\end{aligned}
$$


Here, $T^{-1} A T$ is of (real) block diagonal form. Every block of $T^{-1} A T$ corresponds with an eigenvalue pair of $A$. If the eigenvalue of $A$ is complex, then the block size of the associated block in $T^{-1} A T$ is 2 , if the eigenvalue is real, then the block size is 1 . The LU-costs are now reduced to $\frac{2}{3} d^{3}$ and $\frac{16}{3} d^{3}$ for the blocks of size 1 and 2 , respectively. Hairer $\&$ Wanner used this approach in their code RADAU5 [11]. The blocks of the linear system (5) are now decoupled, so that the use of $\sigma$ processors reduces the effective costs to $\frac{16}{3} d^{3}$, where $\sigma$ is the number of blocks in $T^{-1} A T$. Notice that pairs of stage values can be computed concurrently, i.e. it is possible to do function evaluations, transformations and vector updates for pairs of stages in parallel if $\sigma$ processors are available.

By exploiting the special structure of the $2 d$-dimensional linear systems in (5), it is possible to reduce the costs of solving these systems (see, e.g., [1]). Let $\xi_{j} \pm \mathrm{i} \eta_{j}$ be an eigenvalue pair and assume that the matrix of the corresponding linear system is of the form

$$
\left[\begin{array}{rr}
I-\xi_{j} h_{n} J_{n} & -\eta_{j} h_{n} J_{n} \\
\eta_{j} h_{n} J_{n} & I-\xi_{j} h_{n} J_{n}
\end{array}\right]
$$

One easily checks that the inverse of $(6)$ is

$$
\left(I \otimes \Gamma^{-1}\right)\left[\begin{array}{rr}
I-\xi_{j} h_{n} J_{n} & \eta_{j} h_{n} J_{n} \\
-\eta_{j} h_{n} J_{n} & I-\xi_{j} h_{n} J_{n}
\end{array}\right], \quad \Gamma=I-2 \xi_{j} h_{n} J_{n}+\left(\xi_{j}^{2}+\eta_{j}^{2}\right) h_{n}^{2} J_{n}^{2}
$$

Using $\sigma$ processors, the $\mathcal{O}\left(d^{3}\right)$ costs of this approach are $\frac{8}{3} d^{3}\left(2 d^{3}\right.$ for the computation of $J_{n}^{2}$ and $\frac{2}{3} d^{3}$ for the LU-decomposition of $\Gamma$ ). On $\sigma$ processors, and MRK using this implementation strategy is 4 times more expensive in terms of $\mathcal{O}\left(d^{3}\right)$ costs than an BDF, for which we only have to solve linear systems with a matrix of the form $I-h_{n} \beta J_{n}$.

In this paper we reduce the implementational costs of MRKs further by following the approach of [19]. Here, the matrix $A$ is approximated by a matrix $B$ with positive distinct eigenvalues and the iterates $Y_{n}^{(j)}$ in (4) are computed by means of the inner iteration process

$$
\begin{aligned}
& \left(I-B \otimes h_{n} J_{n}\right)\left(Y_{n}^{(j, v)}-Y_{n}^{(j, v-1)}\right)=-\left(I-A \otimes h_{n} J_{n}\right) Y_{n}^{(j, v-1)}+C_{n}^{(j-1)}, \\
& C_{n}^{(j-1)}:=\left(I-A \otimes h_{n} J_{n}\right) Y_{n}^{(j-1)}-R\left(Y_{n}^{(j-1)}\right) .
\end{aligned}
$$

The index $v$ runs from 1 to $r$ and $Y_{n}^{(j, r)}$ is accepted as the solution $Y_{n}^{(j)}$ of the Newton process (4). Furthermore, $Y_{n}^{(j, 0)}=Y_{n}^{(j-1)}$. Since the matrix $B$ in (8) has distinct eigenvalues, applying a similarity transformation $Q$ that diagonalizes $B$, i.e. $B Q=Q D$ where $D$ is a diagonal matrix, leads to:

$$
\begin{aligned}
\left(I-D \otimes h_{n} J_{n}\right)\left(X_{n}^{(j, v)}-X_{n}^{(j, v-1)}\right)= & -\left(I-Q^{-1} A Q \otimes h_{n} J_{n}\right) X_{n}^{(j, v-1)} \\
& +\left(Q^{-1} \otimes I\right) C_{n}^{(j-1)}, \quad v=1, \ldots, r .
\end{aligned}
$$

The system (9) consists of $s$ decoupled systems of dimension $d$ which can be solved in parallel. Every processor computes a stage value. The costs for the LU-decompositions are now reduced to $\frac{2}{3} d^{3}$ on $s$ processors. Notice that in order to ensure the non-singularity of the matrix $\left(I-D \otimes h_{n} J_{n}\right)$ the positiveness of the eigenvalues of $B$ is required. In analogy with [19] we will refer to (8) as PILSMRK, Parallel Linear System solver for Multistep Runge-Kutta methods. The combination of modified Newton and PILSMRK will be called the Newton-PILSMRK method.

We will discuss several strategies to choose $B$ such that the inner iterates in (8) converge quickly to the Newton iterates in (4). Experiments show that, if we apply more than 2 Newton iterations, 
then only 1 inner iteration suffices to find the Newton iterate. This means that in terms of LUdecompositions and Jacobian evaluations a $k$-step, $s$-stage Newton-PILSMRK on $s$ processors is as expensive as a $k$-step BDF on 1 processor, whereas the stability properties of Newton-PILSMRK are better. If both methods perform the same number of function evaluations, then the accuracies delivered by Newton-PILSMRK are also higher than that of BDF. It turns out that the convergence behavior of the inner iteration process becomes better if $k$ increases. In particular, the inner iteration process for MRKs converges faster than that for the one-step RK methods proposed in [19].

The outline of the paper is as follows. Section 2 briefly describes how to determine the MRK parameters. In Section 3 we investigate the convergence of the inner iteration process for several choices of the matrix $B$, and we consider the stability of the overall method in Section 4 . Numerical experiments in Section 5 show the performance of the proposed methods on a number of test problems. Finally, we draw some conclusions in Section 6.

\section{Construction of MRKs}

A large class of multistep Runge-Kutta methods consists of multistep collocation methods, which were first investigated by Guillou and Soulé [8]. Later, Lie and Nørsett [13] considered the MRKs of Gauss type and Hairer and Wanner [10] those of Radau type. In the useful thesis of Schneider [17] on MRKs for stiff ODEs and DAEs a lot of properties of MRKs and further references can be found.

For the convenience of the reader we briefly describe here how one can compute $c, G$ and $A$. Alternative ways of deriving these parameters can be found in $[10,17]$. In a multistep collocation method, the solution is approximated by a so-called collocation polynomial. Given $y^{(n)}, h^{(n)}$ and $c$, we define the collocation polynomial $u(t)$ of degree $s+k-1$ by

$$
\begin{aligned}
& u\left(t_{j}\right)=y_{j}, \quad j=n-k+1, \ldots, n, \\
& u^{\prime}\left(t_{n}+c_{i} h_{n}\right)=f\left(u\left(t_{n}+c_{i} h_{n}\right)\right), \quad i=1, \ldots, s .
\end{aligned}
$$

The stage vector $Y_{n}$ is then given by $\left(u\left(t_{n}+c_{1} h_{n}\right)^{\mathrm{T}}, \ldots, u\left(t_{n}+c_{s} h_{n}\right)^{\mathrm{T}}\right)^{\mathrm{T}}$. In order to compute $u(t)$, we expand it in terms of polynomials $\phi_{i}$ and $\psi_{i}$ of degree $s+k-1$, given by

$$
\begin{aligned}
& \phi_{i}\left(\tau_{j}\right)=\delta_{i j}, \quad j=1, \ldots, k, \quad i=1, \ldots, k, \\
& \phi_{i}^{\prime}\left(c_{j}\right)=0, \quad j=1, \ldots, s, \quad i=1, \ldots, k, \\
& \psi_{i}\left(\tau_{j}\right)=0, \quad j=1, \ldots, k, \quad i=1, \ldots, s, \\
& \psi_{i}^{\prime}\left(c_{j}\right)=\delta_{i j}, \quad j=1, \ldots, s, \quad i=1, \ldots, s .
\end{aligned}
$$

Here, $\delta_{i j}$ denotes the Kronecker tensor, $\tau$ is the dimensionless coordinate $\left(t-t_{n}\right) / h_{n}$ and $\tau_{j}=$ $\left(t_{n-k+j}-t_{n}\right) / h_{n}, j=1, \ldots, k$. In terms of these polynomials the expansion of $u(t)$ is given by

$$
\begin{aligned}
u\left(t_{n}+\tau h\right) & =\sum_{j=1}^{k} \phi_{j}(\tau) y_{n-k+j}+h_{n} \sum_{j=1}^{s} \psi_{j}(\tau) u^{\prime}\left(t_{n}+c_{j} h_{n}\right) \\
& =\sum_{j=1}^{k} \phi_{j}(\tau) y_{n-k+j}+h_{n} \sum_{j=1}^{s} \psi_{j}(\tau) f\left(u\left(t_{n}+c_{j} h_{n}\right)\right),
\end{aligned}
$$


Clearly, the MRK parameters read $G_{i j}=\phi_{j}\left(c_{i}\right), A_{i j}=\psi_{j}\left(c_{i}\right), \alpha_{j}=\phi_{j}(1)$ and $\chi=\psi_{j}(1)$. Notice that the order of the approximations $u\left(t_{n}+c_{i} h_{n}\right)$, the so-called stage order of the MRK, is $s+k-1$.

To construct the polynomials $\phi_{i}(\tau)$ and $\psi_{i}(\tau)$, we expand them as

$$
\phi_{i}(\tau)=\sum_{m=0}^{s+k-1} d_{m, i}^{\phi} \tau^{m} \quad \text { and } \quad \psi_{i}(\tau)=\sum_{m=0}^{s+k-1} d_{m, i}^{\psi} \tau^{m} .
$$

Substituting the first expression into the defining conditions yields

$$
\left[\begin{array}{cccccc}
1 & \tau_{1} & \tau_{1}^{2} & \tau_{1}^{3} & \cdots & \tau_{1}^{s+k-1} \\
\vdots & \vdots & & \vdots & & \vdots \\
1 & \tau_{k} & \tau_{k}^{2} & \tau_{k}^{3} & \cdots & \tau_{k}^{s+k-1} \\
0 & 1 & 2 c_{1} & 3 c_{1}^{2} & \cdots & (s+k-1) c_{1}^{s+k-2} \\
\vdots & \vdots & & \vdots & & \vdots \\
0 & 1 & 2 c_{s} & 3 c_{s}^{2} & \cdots & (s+k-1) c_{s}^{s+k-2}
\end{array}\right]\left(\begin{array}{c}
d_{0, i}^{\phi} \\
\vdots \\
d_{s+k-1, i}^{\phi}
\end{array}\right)=e_{i} .
$$

The matrix of order $s+k$ in (10) will be denoted by $W$. For the polynomials $\psi_{i}(\tau)$ we derive analogously

$$
W\left(\begin{array}{c}
d_{0, i}^{\psi} \\
\vdots \\
d_{s+k-1, i}^{\psi}
\end{array}\right)=e_{k+i} .
$$

To compute the $A$ and the $G$, we evaluate $\phi_{i}(\tau)$ and $\psi_{i}(\tau)$ in $\tau=c_{j}$ for $j=1, \ldots, s$, yielding

$$
\begin{aligned}
& \phi_{i}\left(c_{j}\right)=\left[\begin{array}{llll}
1 & c_{j} & \cdots & c_{j}^{s+k-1}
\end{array}\right] W^{-1} e_{i},
\end{aligned}
$$

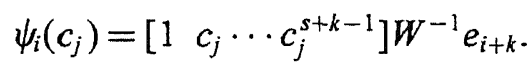

Introducing

$$
V=\left[\begin{array}{cccc}
1 & c_{1} & \cdots & c_{1}^{s+k-1} \\
\vdots & \vdots & & \vdots \\
1 & c_{s} & \cdots & c_{s}^{s+k-1}
\end{array}\right]
$$

the matrices $G$ and $A$ are respectively given by

$$
G=V W^{-1}\left[e_{1} \cdots e_{k}\right] \text { and } A=V W^{-1}\left[e_{k+1} \cdots e_{k+s}\right]
$$

We now construct the abscissae vector $c$ such that we have superconvergence in the step points. Only stiffly accurate Multistep Runge-Kutta methods will be considered, i.e. $c_{s}=1$. This means that we can omit step point formula (2) and obtain $y_{n+1}$ from $y_{n+1}=\left(e_{s}^{\mathrm{T}} \otimes I\right) Y_{n}$. A well known subclass of stiffly accurate MRK methods are the multistep Radau methods, which are $A(\alpha)$-stable. 
Their set of collocation points $c_{1}, \ldots, c_{s-1}$ is given (see $[10$, p. 294]) as the roots in the interval $[0,1]$ of

$$
\sum_{j=1}^{k} \frac{1}{c_{i}-\tau_{j}}+\sum_{\substack{j=1 \\ j \neq i}}^{s} \frac{2}{c_{i}-c_{j}}=0, \quad i=1, \ldots, s-1 .
$$

We call the order of approximation $y_{n+1}$ to $y\left(t_{n+1}\right)$ the step point order or, more loosely, the order of the MRK. This choice of $c$ leads to step point order $2 s+k-2$.

The appendix to this paper lists the MRK parameters for $s \in\{2,4\}$ and $k \in\{2,3\}$.

\section{Convergence of the inner iteration process}

We now discuss the choice of the matrix $B$ in (8) such that the inner iteration process converges rapidly. If we define the inner iteration error by $\varepsilon_{n}^{(j, v)}:=Y_{n}^{(j, v)}-Y_{n}^{(j)}$, then (4) and (8) yield the recursion

$$
\varepsilon_{n}^{(j, v)}=Z\left(h_{n} J_{n}\right) \varepsilon_{n}^{(j, v \sim 1)}, \quad Z\left(h_{n} J_{n}\right):=\left(I-B \otimes h_{n} J_{n}\right)^{-1}\left((A-B) \otimes h_{n} J_{n}\right) .
$$

Applying the method to Dahlquist's test equation

$$
y^{\prime}=\lambda y, \quad \lambda \in \mathbb{C},
$$

this recursion reduces to

$$
\varepsilon_{n}^{(j, v)}=Z\left(z_{n}\right) \varepsilon_{n}^{(j, v-1)}, \quad z_{n}:=h_{n} \lambda .
$$

Let $\mu(\cdot)$ be the logarithmic norm associated with the Euclidean norm, which can be expressed as $\mu(S):=\frac{1}{2} \lambda_{\max }\left(S+S^{\mathrm{T}}\right)$, where $\lambda_{\max }(\cdot)$ denotes the algebraically largest eigenvalue of a matrix (see, e.g., $[9$, p. 61$])$. For dissipative problems $\mu\left(J_{n}\right) \leqslant 0$. The following lemma states that the inner iteration process converges for dissipative problems at least as fast as for the 'most unfavourable' linear test equation. For the proof of this lemma we refer to [15].

Lemma 1. If $\mu\left(J_{n}\right) \leqslant 0$, then $\left\|Z\left(h_{n} J_{n}\right)^{\nu}\right\|_{2} \leqslant \max \left\{\left\|Z^{\nu}\left(z_{n}\right)\right\|_{2}: \operatorname{Re}\left(z_{n}\right) \leqslant 0\right\}$.

In Sections 3.1 and 3.2 we treat two choices for the matrix $B$ that make $Z\left(z_{n}\right)$ 'small' in some sense. To measure $Z\left(z_{n}\right)$ we use the following quantities:

$-\rho^{(j)}\left(z_{n}\right)$, the (averaged) rate of convergence after $j$ iterations in $z_{n}$, defined by

$$
\rho^{(j)}\left(z_{n}\right):=\sqrt[j]{\left\|Z\left(z_{n}\right)^{j}\right\|_{2}}
$$

$-\rho_{\infty}^{(j)}$, the stiff convergence rate after $j$ iterations, defined by

$$
\rho_{\infty}^{(j)}:=\sqrt[j]{\left\|Z_{\infty}^{j}\right\|_{2}}, \quad Z_{\infty}:=\lim _{z_{n} \rightarrow \infty} Z\left(z_{n}\right)=\left(I-B^{-1} A\right) .
$$

$Z_{\infty}$ will be referred to as the stiff amplification matrix. 
Table 1

Values of $\rho^{(j)}$ for several PILSMRK $(L, I)$ methods with constant stepsizes

\begin{tabular}{ccccccccc}
\hline$s$ & $k$ & Order & $j=1$ & $j=2$ & $j=3$ & $j=4$ & $\cdots$ & $j=\infty$ \\
\hline 2 & 1 & 3 & 0.24 & 0.21 & 0.20 & 0.19 & $\ldots$ & 0.18 \\
& 2 & 4 & 0.19 & 0.17 & 0.16 & 0.16 & $\cdots$ & 0.15 \\
& 3 & 5 & 0.17 & 0.15 & 0.15 & 0.14 & $\cdots$ & 0.14 \\
4 & 1 & 7 & 0.59 & 0.54 & 0.53 & 0.52 & $\ldots$ & 0.51 \\
& 2 & 8 & 0.54 & 0.50 & 0.49 & 0.48 & $\ldots$ & 0.47 \\
& 3 & 9 & 0.52 & 0.48 & 0.47 & 0.46 & $\ldots$ & 0.44 \\
8 & 1 & 15 & 1.03 & 0.94 & 0.91 & 0.90 & $\ldots$ & 0.86 \\
& 2 & 16 & 0.98 & 0.92 & 0.89 & 0.88 & $\ldots$ & 0.84 \\
& 3 & 17 & 0.97 & 0.92 & 0.89 & 0.87 & $\cdots$ & 0.82 \\
\hline
\end{tabular}

- $\rho^{(j)}$, the maximal convergence rate after $j$ iterations, defined by

$$
\rho^{(j)}:=\max _{\operatorname{Re}\left(z_{n}\right) \leqslant 0}\left\{\rho^{(j)}\left(z_{n}\right)\right\}
$$

Since all eigenvalues of the matrix $B$ are positive, all the poles of the function $Z(z)$ are in the right-half complex plane. Consequently, $Z^{(j)}(z)$ is analytic in the left-half complex plane and on the imaginary axis. Therefore, we can invoke the maximum principle:

$$
\max _{\operatorname{Re}\left(z_{n}\right) \leqslant 0}\left\|Z^{j}\left(z_{n}\right)\right\|=\max _{x_{n} \geqslant 0}\left\|Z^{j}\left(\mathrm{i} x_{n}\right)\right\| .
$$

(It suffices to confine ourselves to the positive imaginary axis, because $\|Z(z)\|$ is symmetric with respect to the real axis.) Taking the $j$ th square root on both sides, it follows that

$$
\rho^{(j)}=\max _{x_{n} \geqslant 0} \rho^{(j)}\left(\mathrm{i} x_{n}\right)
$$

Since $A$ depends on $h^{(n)}, B$ also depends on $h^{(n)}$. Consequently, the procedure for constructing $B$ has to be carried out every time $h^{(n)}$ changes and should not be too expensive.

\subsection{Constructing B: Crout decomposition}

Let $L$ be the lower triangular matrix of the Crout decomposition of $A$, i.e. $L$ is lower triangular such that $L^{-1} A$ is upper triangular with ones on the diagonal. As proposed in [20], we choose $B=L$. The stiff amplification matrix takes the form $I-L^{-1} A$, which is strictly upper triangular. Consequently, $\rho_{\infty}^{(j)}=0$ for $j \geqslant s$. For reasons that will become clear in Section 3.2, we will refer to this inner iteration process as $\operatorname{PILSMRK}(L, I)$.

Table 1 lists the values of $\rho^{(j)}$ for a few $\operatorname{PILSMRK}(L, I)$ methods for the case with constant stepsizes. As a reference we included the one-step Radau IIA methods. From this table we see that, for the worst-case situation, the convergence of the MRKs is better than that of the one-step Runge-Kutta methods. 


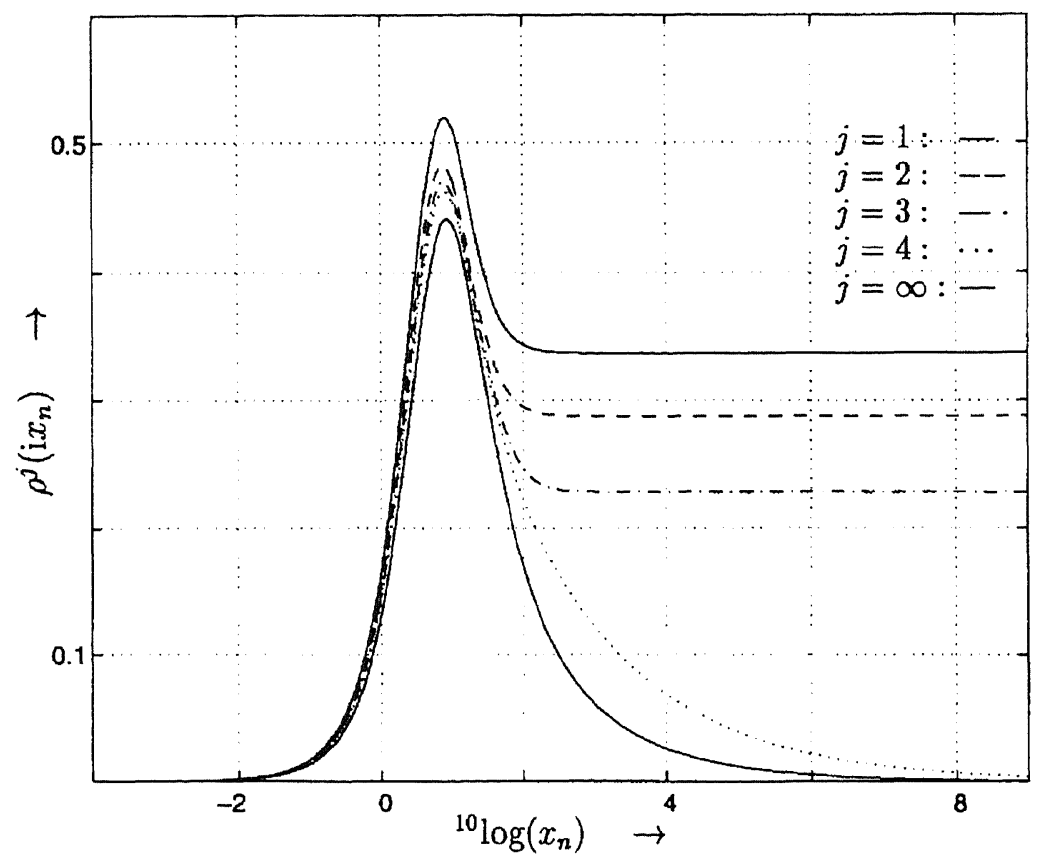

Fig. 1. $\rho^{(j)}\left(\mathrm{i} x_{n}\right)$ for $\operatorname{PILSMRK}(L, I)$ with $k=3, s=4$.

In practice, the rate of convergence in other points of the complex plane is also of interest. Fig. 1 shows $\rho^{(j)}\left(z_{n}\right)$ along the imaginary axis $z_{n}=\mathrm{i} x_{n}, x_{n} \in \mathbb{R}$ for the $\operatorname{PILSMRK}(L, I)$ method with $(k=3, s=4)$ and constant stepsizes for $j=1,2,3,4$ and $j=\infty$. From this figure we clearly see that $\rho_{\infty}^{(j)}=0$ for $j \geqslant s$.

In order to see the effect of variable stepsizes on the convergence rate, we define

$$
\omega_{i}=h_{i} / h_{i-1} \text { for } i=n-k+2, \ldots, n
$$

and plotted $\rho^{(j)}$ as function of $\omega_{i}$ for several PILSMRK methods. Here, $\omega_{i} \in[0.2,2]$, since in an actual implementation, a reasonable factor by which subsequent stepsizes are multiplicated lies in this interval. These plots revealed that the influence of variable stepsizes on the rate of convergence is modest. E.g., for $k=2, s=4, \rho^{(j)} \in[0.45,0.58], \forall j$, and for $k=3, s=4, \rho^{(j)} \in$ $[0.495,0.525], \forall j$.

\subsection{Constructing B: Schur-Crout decomposition}

Before approximating the matrix $A$ by the lower factor of the Crout decomposition, we first transform $A$ to 'a more triangular form', the real Schur form. The next theorem shows that this leads to a damping of the stiff error components that is optimal in some sense. Since most MRKs of interest have matrices $A$ with at most one real eigenvalue, we restrict ourselves to this class. The theorem makes use of the following definition. 
Definition 2. For any $s \times s$ matrix $A$ with at most one real eigenvalue the matrix class $\mathscr{M}_{A}$ consists of matrices $M$ with the property that there exists an orthogonal matrix $U$ such that

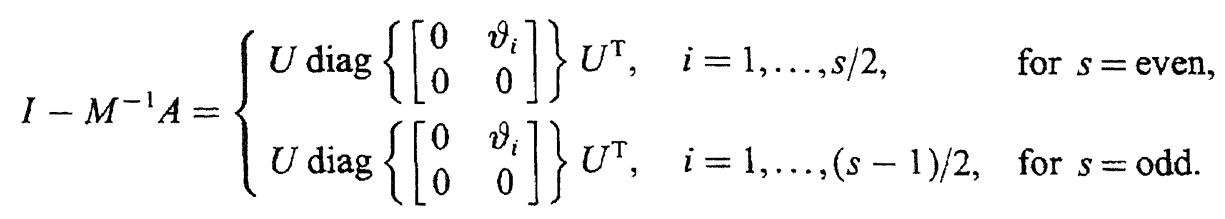

Theorem 3. Let $A$ have at most one real eigenvalue. There can be constructed a matrix $B \in \mathscr{M}_{A}$ for which

(i) $\rho_{\infty}^{(j)}=0$ for $j>1$,

(ii) $\forall M \in \mathscr{M}_{A}: \rho_{\infty}^{(1)} \leqslant\left\|I-M^{-1} A\right\|_{2}$.

Proof. Since there is freedom in the real Schur form of the matrix $A$, we first specify how we construct it. Let $\gamma$ be the vector with eigenvalues of $A$, and $\xi$ and $\eta$ be the real and imaginary part of $\gamma$, respectively, i.e. $\gamma=\xi+$ i $\eta$. Order the components of $\gamma$ as follows (we will motivate this choice later):

$$
\left|\eta_{i}^{2} / \xi_{i}\right| \geqslant\left|\eta_{j}^{2} / \xi_{j}\right| \text { for } i>j
$$

In addition, if $\left|\eta_{i}^{2} / \xi_{i}\right|=\left|\eta_{i+1}^{2} / \xi_{i+1}\right|$, then $\eta_{i}>0$. This sequence is such that, if there is a real eigenvalue, then it has the lowest index in $\gamma$ and complex eigenvalues are ordered in conjugated pairs by increasing value of $\eta_{j}^{2} / \xi_{j}$; the eigenvalue with positive imaginary part comes first within a pair.

Let $e_{j}^{\mathrm{r}}+\mathrm{i} e_{j}^{\mathrm{i}}$ be the eigenvector belonging to $\gamma_{j}$, such that $\left\|e_{j}^{\mathrm{r}}+\mathrm{i} e_{j}^{\mathrm{i}}\right\|_{2}=1$ and $e_{j 1}^{\mathrm{i}}=0$. Define

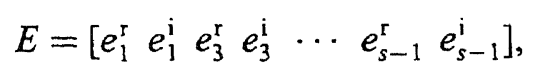

if $A$ has only complex eigenvalues, and

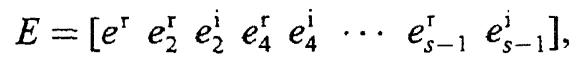

if $A$ has one real eigenvalue with eigenvector $e^{\mathrm{r}}$. One easily verifies that the matrix $E^{-1} A E$ is block diagonal with $2 \times 2$ blocks

$$
\left[\begin{array}{cc}
\xi_{j} & \eta_{j} \\
-\eta_{j} & \xi_{j}
\end{array}\right]
$$

and one block equal to $\xi_{1}$ if $\eta_{1}=0$. We orthonormalize the columns of $E$ by a Gram-Schmidt process, i.e., we construct a lower block triangular matrix $K$ such that $E K$ is orthogonal. This matrix $E K$ transforms $A$ to a matrix $H$ :

$$
H:=(E K)^{-1} A(E K)=K^{-1}\left(E^{-1} A E\right) K \text {. }
$$

Since $K$ is lower triangular and $E^{-1} A E$ is block diagonal, $H$ is lower block triangular. We now rotate the diagonal blocks of $H$ by means of a matrix $\Theta$ given by

$$
\Theta=\operatorname{diag}\left(\Theta_{j}\right), \quad \Theta_{j}=\left[\begin{array}{cc}
\cos \theta_{j} & \sin \theta_{j} \\
-\sin \theta_{j} & \cos \theta_{j}
\end{array}\right], \quad \Theta_{1}=1 \quad \text { if } \eta_{1}=0
$$


Here, $j \in\{2,4, \ldots, s-1\}$ if $\eta_{1}=0$, and $j \in\{1,3, \ldots, s-1\}$ if $\eta_{1} \neq 0$. We will select the angles $\theta_{j}$ such that the second assertion of the theorem will be fulfilled. If we define $S:=\Theta^{-1} H \Theta=U^{\mathrm{T}} A U$, with $U:=E K \Theta$, then $S$ is the desired Schur form of $A$. We denote the lower factor of the Crout decomposition of $S$ by $L$. Setting $B:=U L U^{\mathrm{T}}$ yields a stiff amplification matrix that is similar to $I-L^{-1} S$. Consequently, $B \in \mathscr{M}_{A}$ and $Z_{\infty}^{j}$ vanishes for $j>1$, thereby proving the first part of the theorem.

We choose the parameters $\theta_{j}$ such that $\rho_{\infty}^{(1)}=\max \left\{\left|\vartheta_{j}\right|\right\}$ is minimized. A straightforward analysis shows that

$$
\vartheta_{j}=-S_{j, j+1} / S_{j, j}
$$

where

$$
\begin{aligned}
& S_{j, j}=\frac{1}{2}\left(\left(H_{j, j}-H_{j+1, j+1}\right) \cos \left(2 \theta_{j}\right)+\left(-H_{j, j+1}-H_{j+1, j}\right) \sin \left(2 \theta_{j}\right)+\left(H_{j, j}+H_{j+1, j+1}\right)\right), \\
& S_{j, j+1}=\frac{1}{2}\left(\left(H_{j+1, j}+H_{j, j+1}\right) \cos \left(2 \theta_{j}\right)+\left(H_{j, j}-H_{j+1, j+1}\right) \sin \left(2 \theta_{j}\right)+\left(H_{j, j+1}+H_{j+1, j}\right)\right),
\end{aligned}
$$

and the diagonal blocks of $H$ and $S$ are of the form

$$
\left[\begin{array}{cc}
H_{j, j} & H_{j, j+1} \\
H_{j+1, j} & H_{j+1, j+1}
\end{array}\right] \text { and }\left[\begin{array}{cc}
S_{j, j} & S_{j, j+1} \\
S_{j+1, j} & S_{j+1, j+1}
\end{array}\right] \text {. }
$$

Using Maple [4] we established that $\left|\vartheta_{j}\right|$ is minimized for

$$
\theta_{j}=\arctan \frac{H_{j, j} H_{j+1, j}+H_{j, j+1} H_{j+1, j+1}+\sqrt{\operatorname{det}(H)\left(\|H\|_{F}^{2}-2 \operatorname{det}(H)\right)}}{H_{j+1, j}^{2}+H_{j+1, j+1}^{2}-\operatorname{det}(H)} \bmod \pi,
$$

where $\|\cdot\|_{F}$ denotes the Frobenius norm. Using these values for $\theta_{j}$ in the construction of $B$ leads to the second assertion of the theorem.

\section{Remarks}

- Applying a similarity transformation $Q$ such that $B Q=Q D$, we again arrive at scheme (9). There is freedom in the choice of the transformation matrix $Q$ that diagonalizes $B$. If $X$ is a matrix with eigenvectors of $B$, and $\Sigma$ and $P$ are diagonal and permutation matrices, respectively, then for every matrix $Q$ of the form

$$
Q=X \Sigma P,
$$

we have that $B Q=Q D$. Starting with a fixed matrix $X$, we determine $\Sigma$ and $P$ in (16) such that the elements of $Q$ and $Q^{-1}$ are not too large.

- Unlike for the usual eigenvalue problem, where the eigenvalues and eigenvectors are unknown, here we are faced with the problem of computing a real Schur form, given the eigenvalues and eigenvectors of $A$. The proof serves as a recipe how to construct $B$. We remark that the construction of the real Schur form is not developed to be cheap, but such that we are able to exploit the freedom in the real Schur form.

- Another approach for finding a suitable matrix $B$, based on rotations that minimize $\rho^{(1)}$, can be found in [18]. 
Table 2

Values of $\rho^{(j)}$ for several PILSMRK $(L, U)$ methods with constant stepsizes

\begin{tabular}{ccccccccc}
\hline$s$ & $k$ & Order & $j=1$ & $j=2$ & $j=3$ & $j=4$ & $\cdots$ & $j=\infty$ \\
\hline 2 & 1 & 3 & 0.24 & 0.21 & 0.20 & 0.19 & $\cdots$ & 0.18 \\
& 2 & 4 & 0.18 & 0.16 & 0.16 & 0.15 & $\cdots$ & 0.15 \\
& 3 & 5 & 0.15 & 0.14 & 0.13 & 0.13 & $\cdots$ & 0.13 \\
4 & 1 & 7 & 0.55 & 0.49 & 0.47 & 0.47 & $\ldots$ & 0.44 \\
& 2 & 8 & 0.50 & 0.45 & 0.43 & 0.43 & $\cdots$ & 0.41 \\
& 3 & 9 & 0.47 & 0.42 & 0.41 & 0.40 & $\cdots$ & 0.39 \\
8 & 1 & 15 & 0.91 & 0.78 & 0.74 & 0.72 & $\ldots$ & 0.65 \\
& 2 & 16 & 0.88 & 0.76 & 0.72 & 0.70 & $\cdots$ & 0.62 \\
& 3 & 17 & 0.86 & 0.74 & 0.70 & 0.68 & $\cdots$ & 0.61 \\
\hline
\end{tabular}

- The linear system solver resulting from this Schur-Crout approach will be referred to as PILSMRK $(L, U)$, where the $U$ indicates that we have transformed $A$ before approximating it by $L$.

We now illustrate the idea that moved us to sort the eigenvalues as in (13). For simplicity of notation, we assume here that $s=4$. If the first order expansion of $Z\left(z_{n}\right)$ for small $z_{n}$ is given by

$$
Z\left(z_{n}\right):=z_{n} Z_{0}+\mathcal{O}\left(z_{n}^{2}\right)
$$

then $Z_{0}=A-B$. It can be verified that for the Schur-Crout approach $Z_{0}$ is of the form

$$
Z_{0}=U\left[\begin{array}{cccc}
0 & \zeta_{12} & 0 & 0 \\
0 & \zeta_{22} & 0 & 0 \\
0 & \zeta_{32} & 0 & \zeta_{34} \\
0 & \zeta_{42} & 0 & \zeta_{44}
\end{array}\right] U^{\mathrm{T}}
$$

where

$$
\left(\begin{array}{l}
\zeta_{32} \\
\zeta_{42}
\end{array}\right)=v\left(\begin{array}{c}
S_{31} \\
S_{41}
\end{array}\right), \quad v=-\frac{1}{S_{21}} \frac{\eta_{1}^{2}}{\xi_{1}}
$$

In order to keep the lower triangular part of $Z_{0}$ as small as possible, the best we can do is sorting the eigenvalues such that those with the smallest value of $\eta_{k}^{2} / \xi_{k}$ come first.

Table 2 and Fig. 2 are the analogues of Table 1 and Fig. 1 for $\operatorname{PILSMRK}(L, U)$. In Fig. 2 we clearly see that $\rho_{\infty}^{(j)}$ vanishes for $j>1$. The worst-case $\rho^{(j)}$-values in Table 2 are smaller than those in Table 1. The difference between $\operatorname{PILSMRK}(L, I)$ and $\operatorname{PILSMRK}(L, U)$ becomes larger in favour of $\operatorname{PILSMRK}(L, U)$ as $s$ increases. This can be understood by realizing that for the Crout option, we approximate the matrix $A$ with $s^{2}$ parameters by a matrix $B$ with $\frac{1}{2} s(s+1)$ entries, whereas for the Schur-Crout case, the matrix $U^{\mathrm{T}} A U$ with $\frac{1}{2} s(s+1)+l$ nonzero entries, where $l$ is the number of complex conjugated eigenvalue pairs, is approximated by $U^{\mathrm{T}} B U$ with $\frac{1}{2} s(s+1)$ parameters. The extra price that we have to pay is the construction of the real Schur decomposition of $A$ every time $\omega_{j}$ changes for some $j$. Since in practice $s \ll d$, we do not consider this as a serious drawback.

The matrices $D$ and $Q$ that result from the Crout and Schur-Crout approaches are given in the appendix to this paper for several values of $k$ and $s$. 


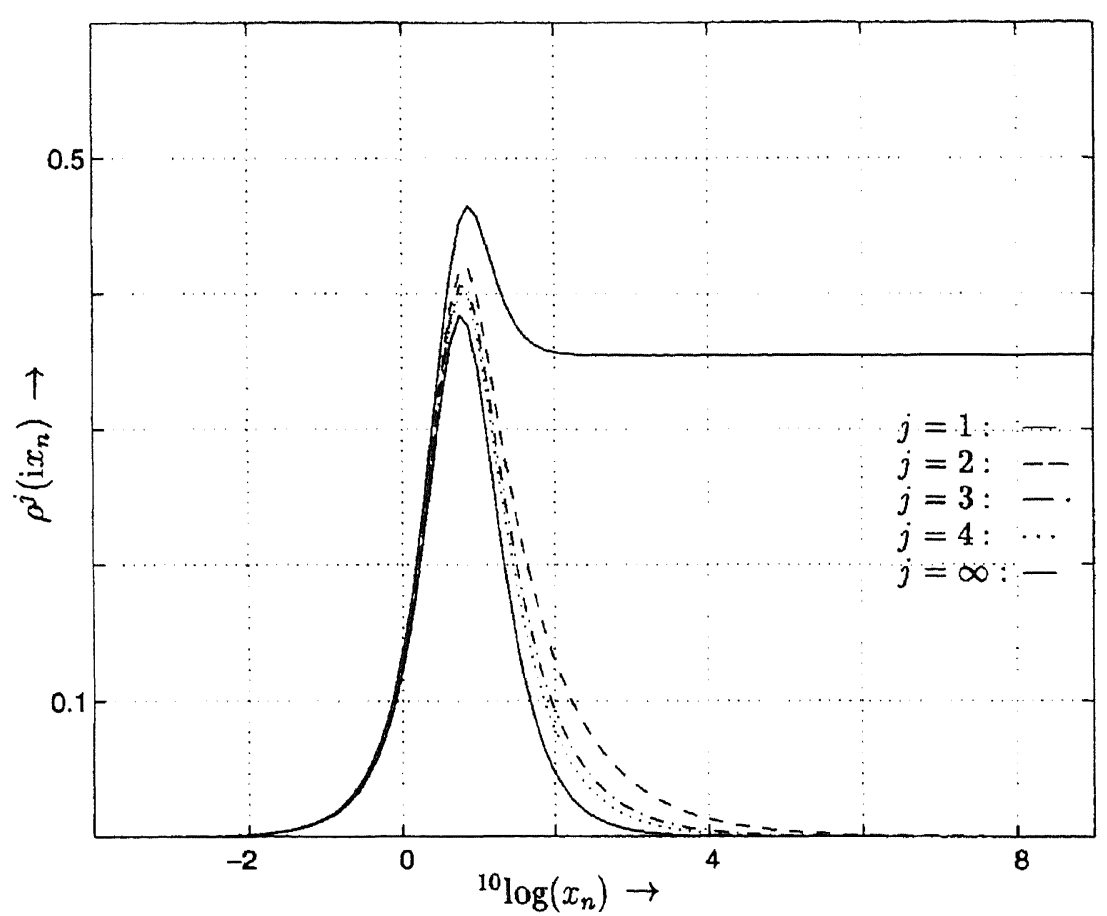

Fig. 2. $\rho^{(j)}\left(\mathrm{ix}_{n}\right)$ for $\operatorname{PLSMRK}(L, U)$ with $k=3, s=4$.

\section{Stability}

We now investigate the stability of the corrector formula (3) and the PILSMRK method given by (8) for test equation (11) solved with constant stepsizes $h$. We only consider stiffly accurate methods, i.e., $y_{n}=\left(e_{s}^{\mathrm{T}} \otimes I\right) Y_{n}^{(m, r)}$.

Following [17], we write (3) in the form

$$
y^{(n)}=M(z) y^{(n-1)}, \quad M(z)=\left[\begin{array}{c}
N \\
e_{s}^{\mathrm{T}}(I-z A)^{-1} G
\end{array}\right], \quad z:=h \lambda,
$$

where the $(k-1) \times k$ matrix $N$ is given by

$$
N=\left[\begin{array}{ccccc}
0 & 1 & 0 & \cdots & 0 \\
\vdots & \ddots & \ddots & \ddots & \vdots \\
\vdots & & \ddots & 1 & 0 \\
0 & \cdots & \cdots & 0 & 1
\end{array}\right] .
$$

The stability region is defined by

$$
\mathscr{S}:=\{z \in \mathbb{C} \mid \rho(M(z))<1\},
$$


where $\rho(\cdot)$ denotes the spectral radius function. We use the quantity $\widehat{D}^{(m r)}$ to measure the stability region (see $[10$, p. 268]), where

$$
\widehat{D}:=-\inf \{\operatorname{Re}(z) \mid z \notin \mathscr{S}\} .
$$

In practice, the PILSMRK method will be used to solve the corrector only approximately. Therefore we do not attain the stability of the corrector. For conducting a stability analysis for the PILSMRK methods we assume that in each step $m$ outer and $r$ inner iterations are carried out. In addition we assume that the predictor is only based on the stage vector in the previous step point,

$$
Y_{n}^{(0, r)}=(P \otimes I) Y_{n-1}^{(m, r)},
$$

where $P$ is an $s \times s$ matrix. From (12) and (3) we derive a recursion in $v$ :

$$
Y_{n}^{(j, v)}=Z(z) Y_{n}^{(j, v-1)}+(I-z B)^{-1} G y^{(n-1)} .
$$

An elementary manipulation, in which we use $Y_{n}^{(j, 0)}=Y_{n}^{(j-1, r)}$, leads to a recursion in $j$ :

$$
Y_{n}^{(j, r)}=Z^{r}(z) Y_{n}^{(j-1, r)}+\left(I-Z^{r}(z)\right)(I-z A)^{-1} G y^{(n-1)} .
$$

Substituting (18) yields the following recursion in time:

$$
Y_{n}^{(m, r)}=Z^{m r}(z) P Y_{n-1}^{(m, r)}+\left(I-Z^{m r}(z)\right)(I-z A)^{-1} G y^{(n-1)},
$$

which we write in the form

$$
\left(\begin{array}{c}
y^{(n)} \\
Y_{n}^{(m, r)}
\end{array}\right)=M^{(m r)}(z)\left(\begin{array}{c}
y^{(n-1)} \\
Y_{n-1}^{(m, r)}
\end{array}\right), \quad M^{(m r)}(z)=\left[\begin{array}{ll}
M_{11}^{(m r)}(z) & M_{12}^{(m r)}(z) \\
M_{21}^{(m r)}(z) & M_{22}^{(m r)}(z)
\end{array}\right] .
$$

From (19) we see that

$$
M_{21}^{(m r)}(z)=\left(I-Z^{m r}(z)\right)(I-z A)^{-1} G, \quad M_{22}^{(m r)}(z)=Z^{m r}(z) P .
$$

Since we restrict ourselves here to stiffly accurate methods,

$$
M_{11}^{(m r)}=\left[\begin{array}{c}
N \\
e_{s}^{\mathrm{T}} M_{21}^{(m r)}
\end{array}\right], \quad M_{12}^{(m r)}=\left[\begin{array}{c}
O_{k-1, s} \\
e_{s}^{\mathrm{T}} M_{22}^{(m r)}
\end{array}\right],
$$

where $O_{i j}$ denotes an $i \times j$ zero matrix. Notice that this linear stability analysis does not distinguish between outer and inner iterations. In analogy with (17) we define the stability region after $\mathrm{mr}$ iterations by

$$
\mathscr{S}^{(m r)}:=\left\{z \in \mathbb{C} \mid \rho\left(M^{(m r)}(z)\right)<1\right\}
$$

and the stability measure

$$
\widehat{D}^{(m r)}:=-\inf \left\{\operatorname{Re}(z) \mid z \notin \mathscr{S}^{(m r)}\right\} .
$$


Table 3

Values of $\widehat{D}^{(m r)}$ for PILSMRK $(L, I)$ with $k$ steps and $s$ stages

\begin{tabular}{llllllllll}
\hline$s$ & $k$ & $m r=1$ & $m r=2$ & $m r=4$ & $m r=6$ & $m r=8$ & $m r=10$ & $m r=20$ & $m r=\infty$ \\
\hline 2 & 1 & 0 & 0 & 0 & 0 & 0 & 0 & 0 & 0 \\
& 2 & 0 & 0 & 0 & 0 & 0 & 0 & 0 & 0 \\
& 3 & 0 & 0.0094 & 0.0823 & 0.0838 & 0.0838 & 0.0838 & 0.0838 & 0.0838 \\
& 4 & 0 & 0.3435 & 0.4601 & 0.4610 & 0.4610 & 0.4610 & 0.4610 & 0.4610 \\
4 & 1 & $*$ & $*$ & 0 & 0 & 0 & 0 & 0 & 0 \\
& 2 & $*$ & $*$ & 0 & 0 & 0 & 0 & 0 & 0 \\
& 3 & $*$ & $*$ & 0 & 0 & 0.0006 & 0.0021 & 0.0025 & 0.0025 \\
& 4 & $*$ & $*$ & 0 & 0 & 0.0120 & 0.0180 & 0.0192 & 0.0192 \\
& 1 & 0 & 0 & 0.0677 & 0.0480 & 0.0239 & 0.0103 & 0 & 0 \\
& 2 & 0 & 0 & 0.0624 & 0.0405 & 0.0188 & 0.0076 & 0 & 0 \\
& 3 & 0 & 0 & 0.0590 & 0.0363 & 0.0162 & 0.0064 & 0 & 0 \\
& 4 & 0 & 0 & 0.0565 & 0.0335 & 0.0145 & 0.0057 & 0.0004 & 0.0003 \\
\hline
\end{tabular}

Table 4

Values of $\widehat{D}^{(m r)}$ for PILSMRK $(L, U)$ with $k$ steps and $s$ stages

\begin{tabular}{|c|c|c|c|c|c|c|c|c|c|}
\hline$s$ & $k$ & $m r=1$ & $m r=2$ & $m r=4$ & $m r=6$ & $m r=8$ & $m r=10$ & $m r=20$ & $m r=\infty$ \\
\hline \multirow[t]{4}{*}{2} & 1 & 0 & 0 & 0 & 0 & 0 & 0 & 0 & 0 \\
\hline & 2 & 0 & 0 & 0 & 0 & 0 & 0 & 0 & 0 \\
\hline & 3 & 0 & 0.0216 & 0.0827 & 0.0838 & 0.0838 & 0.0838 & 0.0838 & 0.0838 \\
\hline & 4 & 0 & 0.3762 & 0.4605 & 0.4610 & 0.4610 & 0.4610 & 0.4610 & 0.4610 \\
\hline \multirow[t]{4}{*}{4} & 1 & * & * & 0.2214 & 0 & 0 & 0 & 0 & 0 \\
\hline & 2 & * & * & 0.2239 & 0 & 0.0001 & 0 & 0 & 0 \\
\hline & 3 & * & * & 0.2784 & 0 & 0.0031 & 0.0030 & 0.0025 & 0.0025 \\
\hline & 4 & * & $*$ & 0.3474 & 0.0001 & 0.0169 & 0.0194 & 0.0192 & 0.0192 \\
\hline \multirow[t]{4}{*}{8} & 1 & 0 & 0.1060 & 0.0636 & 0.0254 & 0.0212 & 0.0101 & 0 & 0 \\
\hline & 2 & 0 & 0.1056 & 0.0557 & 0.0227 & 0.0179 & 0.0080 & 0 & 0 \\
\hline & 3 & 0 & 0.1051 & 0.0510 & 0.0210 & 0.0161 & 0.0075 & 0 & 0 \\
\hline & 4 & 0 & 0.1046 & 0.0477 & 0.0199 & 0.0152 & 0.0075 & 0.0003 & 0.0003 \\
\hline
\end{tabular}

It is clear that

$$
\lim _{m r \rightarrow \infty} \widehat{D}^{(m r)}=\widehat{D} \text {. }
$$

Tables 3 and 4 list $\widehat{D}^{(m r)}$-values for the $k$-step $s$-stage MRK of Radau type for $k \in\{1,2,3,4\}$ and $s \in\{2,4,8\}$ with $\operatorname{PILSMRK}(L, I)$ and $\operatorname{PILSMRK}(L, U)$, respectively. For $s \leqslant 4$, we used the predictor that extrapolates the previous stage values, i.e. we determined $P$ in (18) such that $Y_{n}^{(0, r)}$ has maximal order. Since extrapolating 8 stages leads to very large entries in $P$, the predictor for the 8 -stage methods was chosen to be the last step value predictor. If $\widehat{D}^{(m r)}>4$, then this is indicated by $*$. 


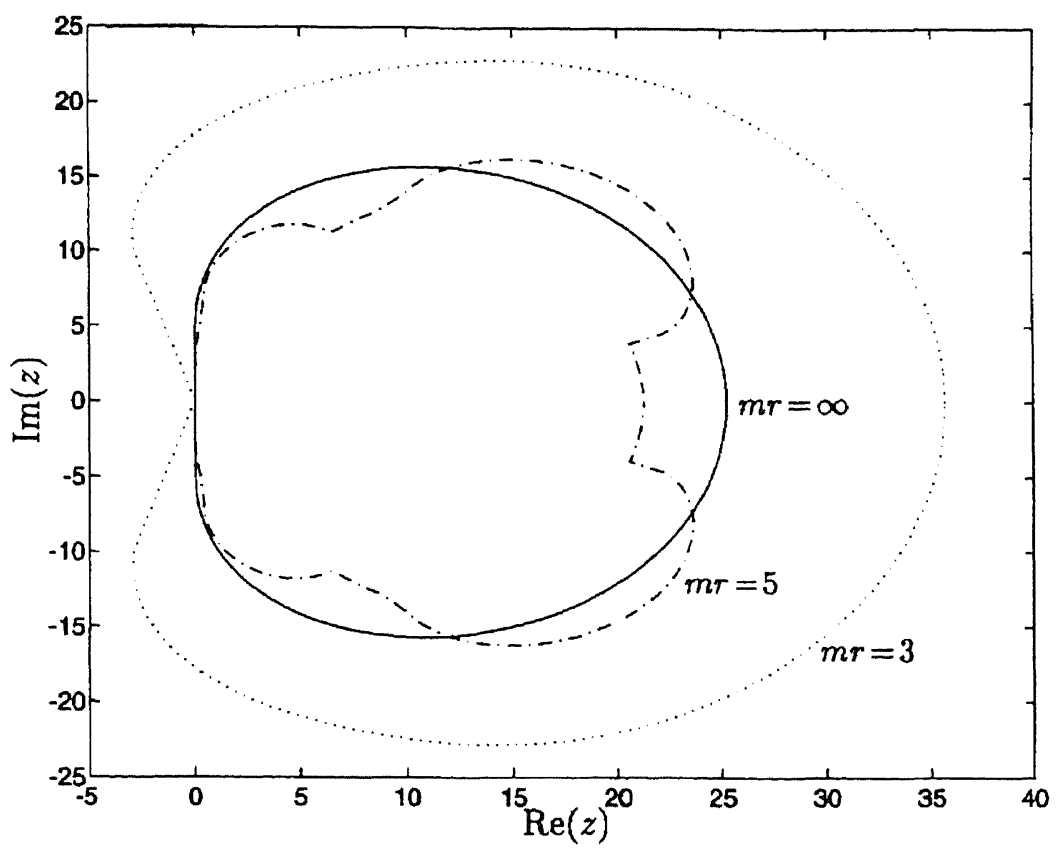

Fig. 3. $\mathscr{S}^{(m r)}$ for $\operatorname{PILSMRK}(L, U)$ with $k=3, s=4$.

The $\widehat{D}^{(m r)}$-values for BDF are independent of $m r$, because for the linear test problem the corrector equation is solved within 1 iteration. For $k=1,2,3$ and 4 these values are $0,0,0.0833$ and 0.6665 , respectively.

From these tables we see that for $s \leqslant 4$ the stability of $\operatorname{PILSMRK}(L, I)$ is better than that of $\operatorname{PILSMRK}(L, U)$. For $s=8$ the $\widehat{D}$-values are comparable. Relatively to its order, the stability of PILSMRK is much better than that of BDF. As expected, we see that increasing $s$ and decreasing $k$ improves the stability of MRK. If we solve the corrector equation only approximately, then sometimes the stability of the resulting method is even better than that of MRK. For $s=4$ and $m r \leqslant 2$, the method is not stable, due to the extrapolation predictor, which is very unstable as stand-alone method. Notice that the $\widehat{D}^{(\infty)}$-values are the values for the underlying MRK corrector.

To get an idea of the shape of $\mathscr{S}^{(m r)}$, Fig. 3 shows $\mathscr{S}^{(m r)}$ for $\operatorname{PILSMRK}(L, U)$ with 3 steps and 4 stages, where $m r \in\{3,5, \infty\}$.

\section{Numerical experiments}

In this paragraph we study the performance of Newton-PILSMRK numerically for more difficult problems then the linear test problem. We conduct three types of experiments. Firstly, we investigate how many inner iterations PILSMRK needs to find the Newton iterate. For this objective, we implement Newton-PILSMRK with fixed stepsizes, a fixed number of Newton and PILSMRK iterations per step and fixed values of $s$ and $k$. 
Secondly, we compare the Newton-PILSMRK method with a BDF formula using modified Newton. Since we expect that both methods will benefit to the same extent from control strategies (i.e. dynamic Newton iteration strategy, stepsize control, etc.), we again perform this experiment using fixed values of $h, s, k, r$ and $m$. For a comparison of MRK codes with one-step Runge-Kutta codes and BDF codes, we refer to Schneider [17], who gives an excellent overview of this subject.

Finally, the parallel performance of Newton-PILSMRK will be investigated.

Two problems from the 'Test Set for IVP Solvers' [14] are integrated. Our first test example is a problem of Schäfer (called the HIRES problem in [10, p. 157]) and consists of 8 mildly-stiff non-linear equations on the interval $[5,305]$. (We adapted the initial condition here such that the integration starts outside the transient phase.) We used stepsize $h=15$. The second test problem originates form circuit analysis and describes a ring modulator. We integrate this highly stiff system of 15 equations on the interval $\left[0,10^{-3}\right]$ with stepsize $h=2.5 \times 10^{-7}$. Horneber [12] provided this problem.

For $s>1$ we implemented the extrapolation predictor as defined before, i.e. based on the previous stage vector. For BDF we used the last step point value as predictor. We tried extrapolation of more step points, but this did not give satisfactory results for both test problems. The starting values $y_{1}, y_{2}, \ldots, y_{k-1}$ were obtained using the 8-stage Radau IIA method, in order to be sure that the integration is not influenced by some starting procedure. In the implementation of BDF we solved the nonlinear equation of dimension $d$ with modified Newton, using $m$ iterations per time step.

In the tables we list the minimal number of correct digits $\mathrm{cd}$ of the components of the numerical solution in the endpoint, i.e. at the endpoint, the absolute errors are written as $10^{-\mathrm{cd}}$. Negative cdvalues are indicated with $*$. The numbers of stages, steps, inner and outer iterations are given by $s, k, r$ and $m$, respectively.

Tables 5 and 6 show that the PILSMRK iterates for $r=1$ are (almost) of the same quality as the Newton iterates, provided that we perform more than 2 Newton iterations.

We also see that the performance of $\operatorname{PILSMRK}(L, U)$ is comparable to that of the $(L, I)$ variant. Although $\operatorname{PILSMRK}(L, U)$ converges faster than $\operatorname{PILSMRK}(L, I)$, the latter has better stability properties for $s \leqslant 4$. Apparently, these effects neutralize each other for these test problems. However, Tables 1-4 indicate that $\operatorname{PILSMRK}(L, U)$ can become better than $\operatorname{PILSMRK}(L, I)$ for $s>4$.

For the 4-stage Newton-PILSMRK, the $k=3$ results are not better than the $k=2$ results. Performing not more then 10 Newton iterations, which is not sufficient to solve the corrector equation, is responsible for this. Experiments confirmed that using more than 10 iterations for the 3-step 4-stage IRK yields higher accuracies than for the 2-step 4-stage method.

From comparing Table 7 with Tables 5 and 6 we learn that Newton-PILSMRK reaches higher accuracies than BDF for the same number of Newton iterations. However, if we want to solve the corrector equation entirely, one would have to perform more Newton iterations for NewtonPILSMRK than for BDF, since the latter is of lower order. Solving the ring modulator, BDF suffers from stability problems for $k=6$, whereas the methods with $k \leqslant 4$ give cd-values, that might be too low in practice.

For a fair comparison of BDF with the new method one should take into account the costs of the Butcher transformations as well. Experiments in [20, Table 4.4], show that for the ring modulator problem, these costs are less than $10 \%$. Since the sequential costs on $s$ processors are $\mathcal{O}(s d)$ for the transformation costs, $\mathcal{O}\left(d^{2}\right)$ for the forward-back substitutions and $\mathcal{O}\left(d^{3}\right)$ for the LU-decompositions, we expect that the contribution of the transformation costs will rapidly decrease for larger problem 
Table 5

Results of PILSMRK $(L, I)$ on test problems

\begin{tabular}{|c|c|c|c|c|c|c|c|c|c|c|c|c|}
\hline$s$ & $k$ & $r$ & $\begin{array}{l}\text { HIRES } \\
m=1\end{array}$ & $m=2$ & $m=3$ & $m=4$ & $m=10$ & $\begin{array}{l}\text { Ring } \\
m=1\end{array}$ & $\begin{array}{l}\text { lator } \\
m=2\end{array}$ & $m=3$ & $m=4$ & $m=10$ \\
\hline \multirow[t]{6}{*}{2} & \multirow[t]{3}{*}{2} & 1 & 3.3 & 3.7 & 4.2 & 4.7 & 4.9 & $*$ & 2.8 & 3.9 & 3.8 & 3.8 \\
\hline & & 2 & 3.2 & 3.8 & 4.3 & 5.0 & 4.9 & $*$ & 3.6 & 3.8 & 3.8 & 3.8 \\
\hline & & 10 & 3.2 & 3.8 & 4.3 & 5.0 & 4.9 & $*$ & 3.6 & 3.8 & 3.8 & 3.8 \\
\hline & \multirow[t]{3}{*}{3} & 1 & 3.3 & 3.7 & 4.2 & 4.6 & 5.2 & $*$ & 3.0 & 4.1 & 4.2 & 4.3 \\
\hline & & 2 & 3.2 & 3.8 & 4.3 & 4.8 & 5.2 & $*$ & 3.8 & 4.2 & 4.3 & 4.3 \\
\hline & & 10 & 3.2 & 3.8 & 4.3 & 4.8 & 5.2 & $*$ & 3.8 & 4.2 & 4.3 & 4.3 \\
\hline \multirow[t]{6}{*}{4} & \multirow[t]{3}{*}{2} & 1 & $*$ & 4.6 & 4.8 & 5.1 & 7.3 & * & $*$ & 6.1 & 6.5 & 8.2 \\
\hline & & 2 & $*$ & 4.3 & 4.9 & 5.3 & 7.9 & * & $*$ & 5.8 & 6.5 & 8.2 \\
\hline & & 10 & 3.7 & 4.4 & 4.9 & 5.4 & 7.9 & * & $*$ & 5.8 & 6.4 & 8.2 \\
\hline & \multirow[t]{3}{*}{3} & 1 & $*$ & 4.6 & 4.8 & 5.1 & 7.2 & $*$ & * & 6.1 & 6.5 & 8.1 \\
\hline & & 2 & $*$ & 4.3 & 4.9 & 5.3 & 7.8 & $*$ & * & 5.8 & 6.5 & 8.1 \\
\hline & & 10 & 3.7 & 4.4 & 4.9 & 5.4 & 7.8 & * & * & 5.8 & 6.4 & 8.1 \\
\hline
\end{tabular}


Table 6

Results of $\operatorname{PILSMRK}(L, U)$ on test problems

\begin{tabular}{|c|c|c|c|c|c|c|c|c|c|c|c|c|}
\hline$s$ & $k$ & $r$ & $\begin{array}{l}\text { HIRES } \\
m=1\end{array}$ & $m=2$ & $m=3$ & $m=4$ & $m=10$ & $\begin{array}{l}\text { Ring } \\
m=1\end{array}$ & $\begin{array}{l}\text { lator } \\
\qquad m=2\end{array}$ & $m=3$ & $m=4$ & $m=10$ \\
\hline \multirow[t]{6}{*}{2} & 2 & 1 & 3.3 & 3.8 & 4.2 & 4.8 & 4.9 & * & 2.8 & 3.9 & 3.8 & 3.8 \\
\hline & & 2 & 3.2 & 3.8 & 4.3 & 5.0 & 4.9 & $*$ & 3.6 & 3.8 & 3.8 & 3.8 \\
\hline & & 10 & 3.2 & 3.8 & 4.3 & 5.0 & 4.9 & * & 3.6 & 3.8 & 3.8 & 3.8 \\
\hline & 3 & 1 & 3.3 & 3.8 & 4.2 & 4.7 & 5.2 & $*$ & 3.1 & 4.1 & 4.3 & 4.3 \\
\hline & & 2 & 3.2 & 3.8 & 4.3 & 4.8 & 5.2 & $*$ & 3.8 & 4.2 & 4.3 & 4.3 \\
\hline & & 10 & 3.2 & 3.8 & 4.3 & 4.8 & 5.2 & $*$ & 3.8 & 4.2 & 4.3 & 4.3 \\
\hline \multirow[t]{6}{*}{4} & 2 & 1 & * & $*$ & 4.9 & 5.1 & 7.2 & * & * & 5.8 & 6.3 & 8.2 \\
\hline & & 2 & 2.6 & 4.4 & 4.9 & 5.4 & 7.9 & * & $*$ & 5.8 & 6.4 & 8.2 \\
\hline & & 10 & 3.7 & 4.4 & 4.9 & 5.4 & 7.9 & $*$ & * & 5.8 & 6.4 & 8.2 \\
\hline & 3 & 1 & $*$ & $*$ & 4.9 & 5.2 & 7.2 & $*$ & $*$ & 5.8 & 6.3 & 8.1 \\
\hline & & 2 & 3.6 & 4.4 & 4.9 & 5.4 & 7.8 & $*$ & * & 5.8 & 6.4 & 8.1 \\
\hline & & 10 & 3.7 & 4.4 & 4.9 & 5.4 & 7.8 & * & $*$ & 5.8 & 6.4 & 8.1 \\
\hline
\end{tabular}

Table 7

Results of BDF on test problems

\begin{tabular}{|c|c|c|c|c|c|c|c|c|c|c|c|c|}
\hline$s$ & $k$ & $r$ & $\begin{array}{l}\text { HIRES } \\
m=1\end{array}$ & $m=2$ & $m=3$ & $m=4$ & $m=10$ & $\begin{array}{l}\text { Ring } \\
m=1\end{array}$ & $\begin{array}{l}\text { lator } \\
m=2\end{array}$ & $m=3$ & $m=4$ & $m=10$ \\
\hline \multirow[t]{5}{*}{1} & 2 & 1 & 2.9 & 3.5 & 3.1 & 3.0 & 3.0 & 1.1 & 1.1 & 1.1 & 1.1 & 1.1 \\
\hline & 3 & 1 & 2.8 & 3.7 & 3.6 & 3.4 & 3.3 & 1.6 & 1.5 & 1.6 & 1.6 & 1.6 \\
\hline & 4 & 1 & 2.8 & 3.4 & 4.4 & 3.8 & 3.6 & 1.8 & 1.9 & 1.9 & 1.9 & 1.9 \\
\hline & 5 & 1 & 2.7 & 3.3 & 4.2 & 4.1 & 3.8 & 2.4 & 2.9 & 2.9 & 2.9 & 2.9 \\
\hline & 6 & 1 & 2.8 & 3.4 & 4.1 & 3.9 & 3.7 & 2.4 & * & 2.9 & * & 2.9 \\
\hline
\end{tabular}


Table 8

Speed-up factor of 3-step 4-stage Newton-PILSMRK $(L, I)$

for ring modulator

\begin{tabular}{llll}
\hline & $m=3$ & $m=4$ & $m=10$ \\
\hline Actual speed-up & 3.3 & 3.3 & 3.2 \\
Optimal speed-up & 3.9 & 3.9 & 3.9 \\
\hline
\end{tabular}

dimensions. For tests of the behavior of the linear algebra part on ODEs of higher dimension, we refer to [7], which studies the linear algebra costs as function of the problem dimension (up to dimension 660) for a method that is comparable to PILSMRK in terms of the solution of linear systems.

In order to show how the Newton-PILSMRK method performs on an $s$-processor computer, we implemented the 3-step 4-stage Newton-PILSMRK $(L, I)$ on the Cray C98/4256 and integrated the ring modulator, using again 4000 constant integration steps. The Cray C98/4256 is a shared memory computer with four processors. Table 8 lists the speed-up factors of the runs on four processors with respect to the runs in one-processor mode. Since we did not have the machine in dedicated mode during our experiments (on the average we used 2.5 processors concurrently), we used a tool called ATExpert [6] to predict the actual speed-up factors on four processors. In practice these values turn out to be very reliable. Denoting the fraction of the code that can be done in parallel by $f_{P}$, the optimal speed-up on $N$ processors according to Amdahl's law is given by the formula $1 /\left(1-f_{P}+f_{P} / N\right)$. ATExpert produces these optimal speed-up values, based on estimates of the parallel fraction $f_{P}$. These values are also listed in Table 8 .

We compiled the codes using the flags $-\mathrm{dp},-\mathrm{ZP}$ and - Wu" $-\mathrm{p} "$ ". The environment variables NCPUS and MP DEDICATED were valued 4 and 1 , respectively. We refer to the Cray C90 documentation [5] for the specification of these settings.

From Table 8 we conclude that the Newton-PILSMRK methods have a satisfactory parallel performance. With respect to the scalability of the method, we remark that the number of processors involved equals the number of stages $s$. Since the step point order is given by $2 s+k-2$, using more than four processors leads to an order that might be too high for most practical applications. Therefore, we aim at two or four processors, which are natural numbers for many computer architectures.

\section{Summary and conclusions}

In this paper we proposed the Newton-PILSMRK method, which is a combination of a Newton process applied to a Multistep Runge-Kutta method with a Parallel Iterative Linear System solver. The non-linear equations that arise in an MRK are usually solved by a modified Newton process, in which we have to solve linear systems of dimension $s d$, where $s$ is the number of Runge-Kutta stages of the MRK and $d$ the dimension of the problem. PILSMRK computes the solutions of these linear systems by means of an inner iteration process, in which we solve $s$ decoupled systems of dimension $d$. To achieve this decoupling, we have to approximate a matrix $A$ with complex eigenvalues by a matrix $B$ with positive distinct eigenvalues. It turns out that: 
- The most efficient parallel implementation of an MRK with a Newton process is 4 times more expensive than Newton-PILSMRK on $s$ processors in terms of $\mathcal{O}\left(d^{3}\right)$ costs.

- If we apply more than 2 Newton iterations, then in practice PILSMRK with only 1 inner iteration often suffices to find the Newton iterate.

- In terms of Jacobian evaluations and LU-decompositions, the $k$-step $s$-stage Newton-PILSMRK on $s$ processors is equally expensive as the $k$-step BDF on 1 processor, whereas the order is higher and the stability properties are better than that of BDF.

- Tests with implementations of Newton-PILSMRK and BDF without control strategies on two problems from the CWI test set show that for the same number of sequential function evaluations, Newton-PILSMRK delivers higher accuracies than BDF, although Newton-PILSMRK did not solve the corrector equation entirely.

- Increasing the number of previous step points $k$, leads to a better convergence behavior of PILSMRK, but worse stability properties of the MRK.

- In a linear stability analysis, performing more than 3 iterations (inner or outer) suffices to attain at least the stability of the MRK corrector, if $s \leqslant 4$.

- Of the two options proposed here for choosing the matrix $B$, Crout and Schur-Crout, the latter has a better convergence behavior, but its stability properties are worse for $s \leqslant 4$.

\section{Acknowledgements}

The authors are grateful to Prof. Dr. P.J. van der Houwen for his careful reading of the manuscript and for suggesting several improvements.

\section{Appendix}

In this appendix we list the parameters $c, G$ and $A$ in (3) for the $k$-step $s$-stage MRK method of Radau type for $k \in\{2,3\}$ and $s \in\{2,4\}$. Moreover, we provide the PILSMRK parameters $\delta$ and $Q$, where $\delta=\operatorname{diag}(D)$ and $D, Q$ are the matrices in (9), for both the Crout approach $\operatorname{PILSMRK}(L, I)$ and the Schur-Crout approach $\operatorname{PILSMRK}(L, U)$. $s=2, k=2$ :

$$
\begin{aligned}
& c^{\mathrm{T}}=\left[\begin{array}{ll}
0.39038820320221 & 1.00000000000000
\end{array}\right], \\
& G=\left[\begin{array}{lr}
-0.04671554852736 & 1.04671554852736 \\
-0.02010509586877 & 1.02010509586877
\end{array}\right], \\
& A=\left[\begin{array}{rr}
0.40044075113659 & -0.05676809646175 \\
0.77072385847003 & 0.20917104566120
\end{array}\right] .
\end{aligned}
$$

Crout:

$$
\begin{aligned}
& \delta^{\mathrm{T}}=\left[\begin{array}{ll}
0.40044075113659 & 0.31843196932797
\end{array}\right] \\
& Q=\left[\begin{array}{ll}
1.00000000000000 & 0 \\
9.39806495685529 & 1.00000000000000
\end{array}\right] .
\end{aligned}
$$


Schur-Crout:

$$
\begin{aligned}
\delta^{\mathrm{T}} & =\left[\begin{array}{ll}
0.36028586267747 & 0.35392212182843
\end{array}\right], \\
Q & =\left[\begin{array}{ll}
0.06418485435680 & 0.05604152383747 \\
0.99793802636797 & 0.99842843890084
\end{array}\right] . \\
S=2, k & =3: \\
c^{\mathrm{T}} & =\left[\begin{array}{ll}
0.42408624230810 & 1.00000000000000
\end{array}\right], \\
G & =\left[\begin{array}{lll}
0.01290709720739 & -0.10843463813621 & 1.09552754092881 \\
0.00354588047065 & -0.04623386039657 & 1.04268797992593
\end{array}\right], \\
A & =\left[\begin{array}{ll}
0.38745055226697 & -0.04598475368028 \\
0.77239469511979 & 0.18846320542493
\end{array}\right] .
\end{aligned}
$$

Crout:

$$
\begin{aligned}
& \delta^{\top}=\left[\begin{array}{ll}
0.38745055226697 & 0.28013523838816
\end{array}\right], \\
& Q=\left[\begin{array}{ll}
1.00000000000000 & 0 \\
7.19743219492460 & 1.00000000000000
\end{array}\right] .
\end{aligned}
$$

Schur-Crout:

$$
\begin{aligned}
& \delta^{\mathrm{T}}=\left[\begin{array}{lll}
0.33129449207677 & 0.32761955124138
\end{array}\right], \\
& Q=\left[\begin{array}{ll}
0.08083975113162 & 0.07616492879483 \\
0.99672711141866 & 0.99709523297510
\end{array}\right] \text {. } \\
& s=4, k=2 \text { : } \\
& c^{T}=\left[\begin{array}{llll}
0.09878664634426 & 0.43388702543882 & 0.80169299888049 & 1.00000000000000
\end{array}\right], \\
& G=\left[\begin{array}{rr}
-0.00087353889029 & 1.00087353889029 \\
0.00062121019919 & 0.99937878980081 \\
-0.00032939714868 & 1.00032939714868 \\
-0.00003663563426 & 1.00003663563426
\end{array}\right] \\
& A=\left[\begin{array}{rrrr}
0.11996670457577 & -0.03384322082318 & 0.01835753398261 & -0.00656791028123 \\
0.26010642038045 & 0.20159324902943 & -0.03956525951247 & 0.01237382574059 \\
0.23561500946812 & 0.41088455735437 & 0.17597260265111 & -0.02110856774179 \\
0.24141835002666 & 0.38984924120599 & 0.31101721961059 & 0.05767855352250
\end{array}\right] \text {. }
\end{aligned}
$$

Crout:

$$
\begin{aligned}
\delta^{\mathrm{T}} & =\left[\begin{array}{lllr}
0.10617138884400 & 0.27770096849016 & 0.27497060030028 & 0.1199667045577
\end{array}\right], \\
Q & =\left[\begin{array}{lllr}
0 & 0 & 0 & 0.01481904140434 \\
0 & 0 & 0.00220678551539 & -0.02486729636785 \\
0 & 0.38896861370956 & -0.38581432071631 & 0.05312025222596 \\
1.00000000000000 & 0.92125100681023 & -0.92257381278026 & -0.99816844890362
\end{array}\right] .
\end{aligned}
$$


Schur-Crout:

$$
\begin{aligned}
\delta^{\mathrm{T}} & =\left[\begin{array}{lrrr}
0.17879165196884 & 0.15567835604316 & 0.18725864804630 & 0.18660124038403
\end{array}\right], \\
Q & =\left[\begin{array}{rrrr}
-0.05047735457027 & -0.05698986733483 & 0.04780729735701 & 0.04801402184956 \\
0.17096598389037 & 0.17933373843615 & -0.16592112501907 & -0.16634392504346 \\
-0.15139062605533 & -0.08731023751861 & 0.17251778528806 & 0.17091936180350 \\
-0.97226722014607 & -0.97824766174222 & 0.96975370911955 & 0.96995408348419
\end{array}\right] . \\
s=4, k & =3: \\
c^{\mathrm{T}} & =\left[\begin{array}{llrr}
0.10504182884419 & 0.44825417107884 & 0.80977028814179 & 1.00000000000000
\end{array}\right], \\
G & =\left[\begin{array}{rrrr}
0.00007487445528 & -0.00195646912651 & 1.00188159467123 \\
-0.00007345206497 & 0.00148038414152 & 0.99859306792346 \\
0.00003966973124 & -0.00083011136249 & 1.00079044163125 \\
0.00000077039880 & -0.00008665832447 & 1.00008588792568
\end{array}\right], \\
A & =\left[\begin{array}{lrrr}
0.12388725564952 & -0.03052720746880 & 0.01502960651127 & -0.00515454606376 \\
0.27600575210564 & 0.19832624728391 & -0.03534802573852 & 0.01060367743938 \\
0.24659262259186 & 0.41336961213203 & 0.16850574024079 & -0.01944845872291 \\
0.25397302181219 & 0.39037260118042 & 0.30064393200968 & 0.05492532747083
\end{array}\right] .
\end{aligned}
$$

Crout:

$$
\begin{aligned}
\delta^{\mathrm{T}} & =\left[\begin{array}{lllr}
0.09980104557325 & 0.26112476902731 & 0.26633715617793 & 0.12388725564952
\end{array}\right], \\
Q & =\left[\begin{array}{lllr}
0 & 0 & 0 & 0.01885332656568 \\
0 & 0 & 0.00429974732457 & -0.03652952061848 \\
0 & 0.38485479574542 & 0.39111661588660 & 0.09232717942550 \\
1.00000000000000 & 0.92297713199827 & 0.92033108442036 & 0.99487981090186
\end{array}\right] .
\end{aligned}
$$

Schur-Crout:

$$
\begin{aligned}
\delta^{\mathrm{T}} & =\left[\begin{array}{lrrr}
0.17281106755693 & 0.15348751145786 & 0.18030166423062 & 0.17980311756297
\end{array}\right], \\
Q & =\left[\begin{array}{rrrr}
-0.03747602767829 & -0.04253832729434 & 0.03538720965186 & 0.03552524964325 \\
0.15438230915055 & 0.16281308949598 & -0.14969201305536 & -0.15002487334226 \\
-0.16907928673314 & -0.11582715575719 & 0.18786790085145 & 0.18664755906307 \\
-0.97271467798559 & -0.97891085323893 & 0.97007509938672 & 0.97025418458887
\end{array}\right] .
\end{aligned}
$$

\section{References}

[1] Z. Bing, $A$-stable and $L$-stable block implicit one-block methods, J. Comput. Math. 3 (4) (1985) 328-341.

[2] P.N. Brown, A.C. Hindmarsh, G.D. Byrne, VODE: a variable coefficient ODE solver, August 1992, Available at http://www.netlib.org/ode/vode.f.

[3] J.C. Butcher, On the implementation of implicit Runge-Kutta methods, BIT 16 (1976) 237-240.

[4] B.W. Char, K.O, Geddes, G.H. Gonnet, B.L. Leong, M.B. Monagan, S.M. Watt, Maple V Language Reference Manual, Springer, New York, 1991. 
[5] Cray Research, Inc. CF77 Commands and Directives, SR-3771 6.0 edition, 1994.

[6] Cray Research, Inc. UNICOS Performance Utilities Reference Manual, SR-2040 8.0 edition, 1994.

[7] J.J.B. de Swart, J.G. Blom, Experiences with sparse matrix solvers in parallel of software, Comput. Math. Appl. 31 (9) (1996) 43-55.

[8] A. Guillou, J.L. Soulé, La résolution numérique des problèmes différentiels aux conditions initiales par des méthodes de collocation, R.I.R.O. R-3 (1969) 17-44.

[9] E. Hairer, S.P. Nørsett, G. Wanner, Solving Ordinary Differential Equations I: Nonstiff Problems, 2nd revised ed., Springer, Berlin, 1993.

[10] E. Hairer, G. Wanner, Solving Ordinary Differential Equations II: Stiff and Differential-algebraic Problems, Springer, Berlin, 1991.

[11] E. Hairer, G. Wanner, RADAU5, July 1996, Available at ftp://ftp.unige.ch/pub/doc/math/stiff/ radau5.f.

[12] E.H. Horneber, Analyse nichtlinearer RLCÜ-Netzwerke mit Hilfe der gemischten Potentialfunktion mit einer systematischen Darstellung der Analyse nichtlinearer dynamischer Netzwerke, Ph.D. Thesis, Universität Kaiserslautern, 1976.

[13] I. Lie, S.P. Nørsett, Superconvergence for multistep collocation, Math. Comput. 52 (1989) 65-79.

[14] W.M. Lioen, J.J.B. de Swart, W.A. van der Veen, Test set for IVP solvers, Report NM-R9615, CWI, Amsterdam, 1996, Available at http://www. Cwi.nl/cwi/projects/IVPtestset.

[15] O. Nevanlinna, Matrix valued versions of a result of Von Neumann with an application to time discretization, J. Comput. Appl. Math. 12 \& 13 (1985) 475-489.

[16] L.R. Petzold, DASSL: A Differential/Algebraic System Solver, June 1991, Available at http://www.netlib . org/ode/ddassl.f.

[17] S. Schneider, Intégration de systèmes d'équations différentielles raides et différentielles-algébriques par des méthodes de collocations et méthods générales linéaires, Ph.D. Thesis, Université de Genéve, 1994.

[18] P.J. van der Houwen, E. Messina, Parallel linear system solvers for Runge-Kutta-Nyström methods, Technical Report NM-R9613, CWI, Amsterdam, 1996, submitted for publication.

[19] P.J. van der Houwen, J.J.B. de Swart, Parallel linear system solvers for Runge-Kutta methods, Adv. Comput. Math. 7 (1997) $157-181$.

[20] P.J. van der Houwen, J.J.B. de Swart, Triangularly implicit iteration methods for ODE-IVP solvers, SIAM J. Sci. Comput. 18 (1) (1997) 41-55. 